\title{
Políticas públicas para el adulto mayor en época de pandemia por COVID- 19
}

\author{
Fecha de recepción: 2021-10-29 • Fecha de aceptación: 2021-12-14 • Fecha de publicación: 2022-01-10
}

\author{
María Cristina Espín Meléndez ${ }^{1}$ \\ Universidad Técnica de Ambato, Ecuador \\ mariacespinm@uta.edu.ec \\ https://orcid.org/0000-0002-0966-8272
}

Johana Estefanía Constante Lascano ${ }^{2}$ Universidad Técnica de Ambato, Ecuador johanaconstante76@gmail.com https://orcid.org/0000-0002-1743-0571

Diego Francisco Granja Zurita ${ }^{3}$ Universidad Regional Autónoma de los Andes, Ecuador ua.diegogranja@uniandes.edu.ec https://orcid.org/0000-0002-1305-3895

\section{Resumen}

Los adultos mayores son un grupo de atención prioritaria protegidos por la Constitución de la República del Ecuador, y, por lo tanto, las acciones estatales deberían estar orientadas a la protección de los mismos, para mejorar su calidad de vida. Durante la pandemia COVID-19 fueron el grupo humano más afectado por este virus, por lo que el objeto de este estudio es evidenciar el nivel de protección del Estado a los derechos del adulto mayor en la localidad de Tisaleo. La metodología aplicada fue cuantitativa con un enfoque descriptivo y una modalidad investigativa de campo, la población fueron las personas adultas mayores del cantón Tisaleo de la Provincia de Tungurahua y bajo un muestreo no probabilístico se seleccionó a 80 adultos mayores de 65 años en adelante. Entre los resultados principales se obtuvo que el $75 \%$ tuvo una mala economía durante la pandemia, el $100 \%$ contó con servicios básico como agua y luz, el $85 \%$ tuvo una mala salud, el $45 \%$ padeció 
de artritis y artrosis, la mayor parte de encuestados se alimentó 3 veces al día, el 100\% no contó con actividades de ocio, entre otros resultados. Como parte de las conclusiones se determina que, durante la pandemia, las políticas estatales no beneficiaron significativamente a la calidad de vida del adulto mayor, presentándose deficiencias en el sistema de salud pública, suspensión de programas de ocio y participación, inexistencia de programas alimentarios, viéndose las acciones del Gobierno local dirigidas a prevenir y mitigar los efectos del COVID-19.

Palabras clave: adulto mayor, calidad de vida, políticas públicas, pandemia

\begin{abstract}
Older adults are a priority group protected by the Constitution of the Republic of Ecuador, and therefore, state actions should be oriented to their protection in order to improve their quality of life. During the COVID-19 pandemic, they were the human group most affected by this virus, so the purpose of this study is to demonstrate the level of State protection of the rights of the elderly in the town of Tisaleo. The methodology applied was quantitative with a descriptive approach and a field research modality, the population was the elderly people of the canton of Tisaleo in the Province of Tungurahua and under a non-probabilistic sampling, 80 elderly adults aged 65 years and older were selected. Among the main results were that $75 \%$ had a bad economy during the pandemic, $100 \%$ had basic services such as water and electricity, $85 \%$ had poor health, $45 \%$ suffered from arthritis and arthrosis, most respondents ate 3 times a day, $100 \%$ had no leisure activities, among other results. As part of the conclusions it is determined that, during the pandemic, state policies did not significantly benefit the quality of life of the elderly, presenting deficiencies in the public health system, suspension of leisure and participation programs, non-existence of food programs, seeing the actions of the local government aimed at preventing and mitigating the effects of COVID-19.
\end{abstract}

Keywords: elderly, quality of life, public policies, pandemic 


\section{Introducción}

La pandemia por COVID-19 comenzó a permearse en todo el mundo como una nueva enfermedad aterradora a principios del 2020, se convirtió en una crisis global por su propagación y efectos contagiosos todos los días, así como también, tiene un impacto negativo importante en la salud, la economía, la sociedad y la seguridad de las comunidades (Acuña, 2021). Los Gobiernos nacionales comenzaron a luchar contra la destrucción del sector salud, con cambios repentinos en los campos psicológico, social, económico y político, eligiendo la vida por encima de las economías y una mayor expansión (Rojo \& Bonilla, 2020).

La pandemia hace que la persona presente síntomas graves como una enfermedad de las vías respiratorias y puede complicar la situación de esta según los grupos de edad. Si bien muestra un curso leve de la enfermedad en la población más joven, puede causar síntomas graves y un mayor riesgo de mortalidad, especialmente entre las personas mayores y aquellas con problemas de salud previos (Downes \& Brosseuk, 2021). Como resultado del curso de esta enfermedad y las tasas de mortalidad, muchos países han establecido reglas estrictas para contrarrestar un brote. Además de, las prácticas estrictas, los individuos implementaron sus propias medidas de distanciamiento social, las cuales tuvieron un amplio impacto en la vida familiar y laboral (Flórez, 2020).

A nivel mundial, los adultos mayores fueron identificados desde el principio de la pandemia por COVID-19, como personas de mayor riesgo de enfermarse gravemente por causa del virus, o inclusive que podría conllevarle a la muerte. Las afecciones médicas (asma, diabetes, enfermedades cardíacas u otras) que ocurren con más frecuencia en la edad adulta eran un factor de riesgo (Petrilli, 2020). Por lo establecido, los adultos mayores constituyen un grupo heterogéneo debido a diferentes experiencias de vida, antecedentes culturales, genética, historiales de salud y contextos en los que están envejeciendo.

Durante la pandemia, la salud y el bienestar de los adultos mayores se vieron influidos no solo por el riesgo de contraer el virus o enfermarse, sino por los efectos adversos que conllevan las recomendaciones de distanciamiento social o la preocupación por los familiares, la economía, salud y el bienestar. Limitar las actividades fuera del hogar y la interacción social cambiaron los hábitos y las actividades diarias de los adultos mayores de maneras que de antemano pueden ser difíciles de estimar (García, 2021).

Las autoridades nacionales y locales de los niveles tres de salud han tenido que reaccionar rápidamente para limitar la propagación de la enfermedad y evitar el colapso de los sistemas de suministro de salud. Ecuador, y el mundo en general, vive actualmente una emergencia real que indiscutiblemente juega un papel en la configuración de los cantones, que son los lugares más vulnerables a las emergencias sanitarias, debido a la alta densidad de personas y actividades.

En la gestión de cualquier crisis de salud pública, una ciudad tiene dos tareas generales, a saber, hacer frente al gran número repentino de personas enfermas y mantener la vida en la ciudad lo más normal posible para todos los demás, especialmente para los residentes más vulnerables, como las personas mayores, con el fin de proporcionarles suficiente asistencia sanitaria y social 
(Martínez, 2020). Tanto el COVID-19, como el envejecimiento de la población ecuatoriana, son los principales temas de la investigación, por lo tanto, el objetivo principal de este estudio fue explorar las experiencias vividas de los adultos mayores del cantón Tisaleo de la provincia de Tungurahua, Ecuador, durante la pandemia, para analizar el derecho a la vida.

En el Ecuador, según lo establecido en el año 2021 por Instituto Nacional de Estadísticas y Censos, en el 2025 se evidenciará un significativo aumento de la población adulta mayor con un estimado de 1'592.232 (INEC, 2010), como se evidencia en la Tabla 1.

Tabla 1.

Población de adultos mayores por años

\begin{tabular}{lccc}
\hline Grupos de edad & $\mathbf{2 0 0 1}$ & $\mathbf{2 0 0 6}$ & $\mathbf{2 0 2 5}$ \\
\hline 65 y más & 645.191 & 779.770 & $11^{\prime} 592.232$ \\
\hline
\end{tabular}

Fuente: INEC (2010)

La población adulta mayor representa en la actualidad el 7,2\% del total de la población. El envejecimiento son los cambios fisiológicos progresivos en un organismo que conducen a la senescencia, o una disminución de las funciones biológicas y de la capacidad del organismo para adaptarse al estrés metabólico, por ello, el envejecimiento exitoso se define como un alto funcionamiento físico, psicológico y social en la vejez, sin enfermedades importante (Urtamo et al., 2019).

En consideración a la vida digna, es un derecho constitucional que involucra a todas las personas sin distinción, el cual involucra un nivel y calidad de vida adecuado. El derecho a la vida digna está comprometido con la salud, alimentación, nutrición, derecho al agua, vivienda, saneamiento, educación, trabajo, empleo, descanso y ocio, cultura física, vestido, seguridad social, entre otros, consecuentemente, el Estado es el encargado de eliminar la violencia ejercida en contra de mujeres, niñas, niños y adolescentes, personas adultas mayores, personas con discapacidad, y demás personas que se hallen en una situación de desventaja social o vulnerabilidad (Robalino, 2018).

La calidad de vida bajo un concepto multidimensional, se centra en la percepción que tiene la misma persona, sobre la vida diaria en un sistema de valores y en contextos culturales, asi como también metas, intereses, normas e inquietudes, autonomía, salud, independencia, satisfacción con la vida y aspectos ambientales como redes de apoyo y servicios sociales, entre otros (Martínez, 2020, p. 17-18).

Los adultos mayores tienen derecho a la vida y a una atención preferente adecuada, ya sea por una enfermedad catastrófica, por discapacidad u otra situación, son un grupo poblacional beneficiado, aún no se establece acciones concretas para disminuir el abandono y pobreza (Robalino, 2018). Mejorar la calidad de vida de todas las personas es el principal fin de todo Gobierno, pero suele ser un verdadero reto por cumplir, debido que al ser un grupo desprotegido se vuelven presa fácil de cualquier problema social, en caso de las personas adultas mayores se ven diariamente sometidos a discriminación por edad, condición física, abandono, maltrato y 
violencia, u otros, al especificar el derecho constitucional a la vida digna prescrito en el numeral 2 de artículo 66 en concordancia con el artículo 16 de la Ley Orgánica de las personas adultas mayores.

En el cantón Tisaleo se ha trabajado sobre planes, programas, proyectos, y políticas públicas emitidas antes de la llegada de la pandemia, tales como la política pública "Mis mejores años", que surgió como una iniciativa a fin de mejorar la calidad de vida de las personas de 65 años, en situación de pobreza extrema. En el cantón Tisaleo se dirige esta política pública a aquellas personas adultas mayores que se encuentran en situación de pobreza y pobreza extrema de los barrios o cabildos altos, siendo alrededor de 2000 personas adultas mayores que se encuentran localizadas en el cantón Tisaleo, de los cuales el programa mantiene la cobertura únicamente para 120 de ellas, lo que correspondería un $6 \%$ de cobertura total del proyecto, mientras que el $94 \%$ restante aún esta desprotegido.

Además, se encuentran trabajando con el programa "Grupos Activos", conformado por 50 o 60 personas adultas mayores aproximadamente, pertenecientes a las comunidades de Tisaleo centro, Santa Lucía-La Libertad, Santa Lucía Centro, Alobamba, y Quinchicoto, este plan o programa se mantiene con el convenio del Consejo Provincial de Tungurahua, quienes apoyan con personal capacitado, tales como nutricionistas, técnicos, entre otros, que ayudan de 2 a 3 horas en actividades diversas y programas para su beneficio cada 15 días en los hogares de las personas adultas mayores.

Actualmente, en el cantón Tisaleo se ha desarrollado la "Ordenanza de exoneración del pago del agua potable", dirigido a personas adultas mayores y personas con discapacidad, lo cual se espera su posterior aprobación a fin de ayudar y mejorar la situación de este grupo; así mismo, se aguarda la aprobación del proyecto de creación de un centro gerontológico para la atención y cuidado de las personas adultas mayores, que requieren protección especial y que no pueden ser atendidos por sus familiares, ya que muchos adultos mayores acuden a centros lejanos para recibir esta atención.

\section{Metodología}

El enfoque de la investigación fue cuantitativo mediante la obtención de datos numéricos y estadísticos con un nivel de investigación descriptivo (Hernández-Sampieri \& Mendoza, 2018), con el fin, de describir la situación de los adultos mayores durante la pandemia. La población de estudio fueron las personas adultas mayores del cantón Tisaleo, de la Provincia de Tungurahua, con total de 856 personas bajo un muestreo no probabilístico se seleccionó a 80 adultos mayores de 65 años en adelante para formar parte de la investigación.

Se aplicó una modalidad investigativa de campo debido a que se realizó en el cantón Tisaleo de la Provincia de Tungurahua, mediante la aplicación de encuestas, la misma que estuvo constituida por los siguientes puntos: datos demográficos, economía, servicios básicos, salud, enfermedades, medidas tomadas por el Estado, acceso a médico y medicina, alimentación, actividades de ocio, percepción de calidad de vida y necesidades de las personas adultas. 
Se utilizó la técnica de encuesta para proporcionar a la investigación exactitud y precisión en cuanto a los datos referentes al tema de investigación planteada. El levantamiento de información se realizó en la zona central del cantón, mediante visitas domiciliarias, la información recabada fue procesada y analizada mediante Microsoft Excel.

\section{Resultados}

Para iniciar se toma en consideración los datos demográficos de los adultos mayores, del $100 \%$ de personas encuestadas el 50\% tienen 65 y 70 años, por otra parte, el 31\% tienen 70 y 75 años de edad, en una representación minoritaria, el 10\% se encuentra entre los 75 a 80 años, para finalizar entre 80 a 85 años y de 85 a 90 años se identificó un $6 \%$ y 3\% respectivamente. En consideración al género, el $79 \%$ son de género masculino y el $21 \%$ restantes son de género femenino.

\subsection{Situación del adulto mayor durante la pandemia}

Figura 1.

Economía durante la pandemia

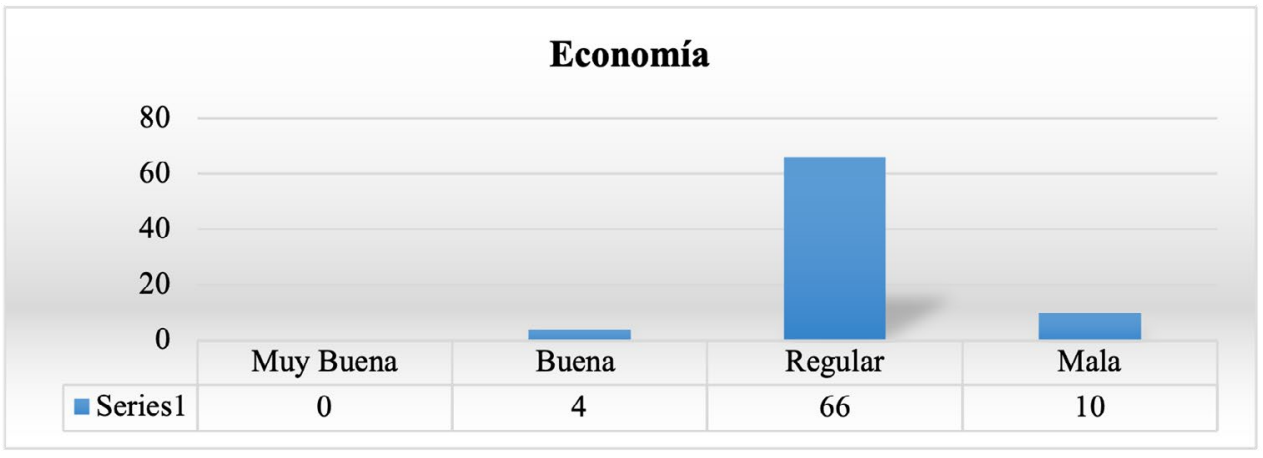

En función a la situación económica del adulto mayor durante la pandemia, se obtuvo que el $75 \%$ mantuvo una económica regular (Figura 1), el 12\% contó con una economía mala y tan solo el $5 \%$ describió que su economía fue buena, es relevante mencionar que no existe ninguna representación de una economía muy buena. Se infiere que este resultado fue ocasionado por la el impacto económico significativo en todo el mundo, debido a la reducción de la productividad, la pérdida de vidas, el cierre de empresas, la interrupción del comercio y la destrucción de la industria del turismo (Pak et al., 2020).

Tabla 2.

Servicios Básicos

\begin{tabular}{lccc}
\hline & Si & No & TOTAL \\
\hline Luz & 80 & 0 & 80 \\
\hline Agua & 80 & 0 & 80 \\
\hline Teléfono & 6 & 74 & 80 \\
\hline Internet & 0 & 80 & 80 \\
\hline
\end{tabular}


Se entiende que, para cumplir con el derecho de vida digna, la población de adultos mayores debe contar con los servicios básicos, por ello, el 100\% de los encuestados tiene disponible luz y agua, por otra parte, el $92 \%$ no dispone de teléfono y el $100 \%$ no cuenta con Internet, como se observa en la Tabla 2.

Tabla 3.

Salud de los adultos mayores

\begin{tabular}{lcc}
\hline Estimación & Frecuencia & $\%$ \\
\hline Muy Buena & 0 & $0 \%$ \\
\hline Buena & 3 & $3.75 \%$ \\
\hline Regular & 68 & $85 \%$ \\
\hline Mala & 9 & $11.25 \%$ \\
\hline TOTAL & 80 & $100 \%$ \\
\hline
\end{tabular}

Por lo tanto, durante la pandemia se consideró a los adultos mayores como un grupo de riesgo. Como respuesta, han aumento las preocupaciones sobre la salud de los adultos mayores. La Organización Mundial de la Salud (OMS) advirtió que el impacto en el bienestar físico, mental y psicosocial de los grupos vulnerables, como los adultos mayores, sería más grande y duradero (De Pue et al., 2021). Bajo la premisa de la opinión de los autores, el estudio evidenció que la salud de los encuestados fue regular en el $85 \%$ durante la pandemia, para el $11 \%$ fue mala y tan solo para el 3\% fue buena (Tabla 3).

Tabla 4.

Enfermedades de las personas adultas mayores

\begin{tabular}{lcc}
\hline Enfermedades & Frecuencia & $\%$ \\
\hline Artritis y Artrosis & 36 & $45 \%$ \\
\hline Dolores de Cabeza & 5 & $6 \%$ \\
\hline Diabetes & 2 & $3 \%$ \\
\hline Dolores musculares & 12 & $15 \%$ \\
\hline Gastritis & 3 & $4 \%$ \\
\hline Gripes comunes & 2 & $3 \%$ \\
\hline Enfermedades pulmonares & 1 & $1 \%$ \\
\hline Cáncer de colon & 1 & $1 \%$ \\
\hline Fractura de Columna & 1 & $1 \%$ \\
\hline Epilepsia & 1 & $1 \%$ \\
\hline Hernias & 1 & $1 \%$ \\
\hline Problemas Cardiacos & 6 & $8 \%$ \\
\hline Enfermedades Prostáticas & 6 & $8 \%$ \\
\hline Ninguna & 3 & $4 \%$ \\
\hline TOTAL & $\mathbf{8 0}$ & $100 \%$ \\
\hline
\end{tabular}


Tomando en cuenta las enfermedades que padecieron los adultos mayores durante la pandemia, el $45 \%$ padeció artritis y artrosis, el $15 \%$ dolores musculares y el $8 \%$ problemas cardiacos y enfermedades prostáticas, existió representaciones minoritarias de otras enfermedades, ver Tabla 4. Autores como Jaul \& Barron (2017), especifican que el proceso de envejecimiento es universal, pero no uniforme, detallan que la conciencia de los cambios fisiológicos relacionados con la edad, como la disminución de la agudeza visual y auditiva, el tiempo de reacción lento y el equilibrio alterado, prepararán a los pacientes y cuidadores para manejar los riesgos, tomar decisiones informadas y quizás prevenir caídas y efectos adversos de los medicamentos. También se conoce que, las enfermedades cardiovasculares y la osteoporosis y la demencia son afecciones crónicas comunes a los 85 años. La osteoartritis, la diabetes y la discapacidad relacionada con la movilidad aumentarán en prevalencia a medida que la población envejezca y tenga más sobrepeso.

Tabla 5.

Medidas tomadas por el Estado

\begin{tabular}{lcc}
\hline Medidas & Frecuencia & $\%$ \\
\hline Brigadas médicas y medicina & 5 & $6 \%$ \\
\hline Vacunas & 32 & $40 \%$ \\
\hline Distanciamiento social & 16 & $20 \%$ \\
\hline Estado de excepción & 1 & $1 \%$ \\
\hline $\begin{array}{l}\text { Restricciones de movilidad y } \\
\text { saneamiento }\end{array}$ & 9 & $11 \%$ \\
\hline Desconoce & 17 & $21 \%$ \\
\hline TOTAL & $\mathbf{8 0}$ & $100 \%$ \\
\hline
\end{tabular}

Las medidas tomadas por el Estado para la población del cantón Tisaleo fueron en el $40 \%$ aplicación de vacunas, en el $20 \%$ establecer medidas para el distanciamiento social y en el $11 \%$ restricción de movilidad y saneamiento (Tabla 5). A nivel mundial, las principales medidas tomadas prevenir la propagación en la sociedad con la higiene de las manos, distanciamiento social y cuarentena. Con una mayor capacidad de prueba, la detección de más pacientes COVID-19 positivos en la comunidad también permitirá la reducción de casos secundarios con reglas de cuarentena más estrictas (Güner et al., 2020).

Tabla 6.

Acceso médico y medicinas

\begin{tabular}{lcc}
\hline Atención medica & Frecuencia & $\%$ \\
\hline Muy Bueno & 0 & $0 \%$ \\
\hline Bueno & 0 & $0 \%$ \\
\hline Regular & 4 & $5 \%$ \\
\hline Malo & 2 & $2 \%$ \\
\hline No acudió & 27 & $34 \%$ \\
\hline Médico Particular & 47 & $59 \%$ \\
\hline TOTAL & 80 & $100 \%$ \\
\hline
\end{tabular}


De acuerdo con la Tabla 6, el acceso a la atención médica y fármacos fue en el 59\% por un médico particular y no por la salud pública, y el 34\% no acudió cuando requirió atención médica.

Tabla 7.

Alimentación

\begin{tabular}{lcc}
\hline Alimentación & Frecuencia & $\%$ \\
\hline 1 vez & 0 & $0 \%$ \\
\hline 2 veces & 0 & $0 \%$ \\
\hline 3 veces & 76 & $95 \%$ \\
\hline 4 veces & 4 & $5 \%$ \\
\hline Más de 4 veces & 0 & $0 \%$ \\
\hline TOTAL & $\mathbf{8 0}$ & $100 \%$ \\
\hline
\end{tabular}

En relación a la alimentación de los adultos mayores, esta fue de 3 veces en el 95\% de los encuestados y el 5\% se alimentaba 4 veces al día. Como menciona McFarland et al. (2013), para los adultos mayores es importante comer con regularidad, al menos tres veces al día.

Tabla 8.

Actividades de ocio

\begin{tabular}{lcc}
\hline Actividades de ocio & Frecuencia & $\%$ \\
\hline Programas & 0 & $0 \%$ \\
\hline Proyectos & 0 & $0 \%$ \\
\hline Actividades diversas & 0 & $0 \%$ \\
\hline Ningún apoyo & 80 & $100 \%$ \\
\hline TOTAL & 80 & $100 \%$ \\
\hline
\end{tabular}

La calidad de vida consta de múltiples aspectos cotidianos, entre los cuales se puede mencionar algunas actividades tales como ocio, vecindario y vida familiar que ocupan la mayor parte de la vida de las personas mayores después de la jubilación. Con tiempo discrecional e ingresos disponibles, las personas mayores están más dispuestas a participar en actividades de ocio y turismo, y el ámbito de la vida de ocio de las personas mayores se convierte en el conducto principal para actividades sociales (Zhang \& Zhang, 2018)this research aims to clarify how leisure and tourism contribute to the elderly's quality of life (QOL. Por lo descrito, en los resultados de la presente investigación se identificó que el 100\% no conto con ningún apoyo para actividades de ocio, como se observa en la Tabla 8.

Tabla 9.

Percepción de calidad de vida

\begin{tabular}{lcc}
\hline Estimación & Frecuencia & $\%$ \\
\hline Muy Buena y feliz & 1 & $1 \%$ \\
\hline
\end{tabular}




\begin{tabular}{lll}
\hline Buena & 11 & $14 \%$ \\
\hline Regular & 19 & $24 \%$ \\
\hline Mala y triste & 49 & $61 \%$ \\
\hline TOTAL & $\mathbf{8 0}$ & $100 \%$ \\
\hline
\end{tabular}

La calidad de vida se utiliza a menudo como un conjunto de indicadores integrales para medir la situación de las personas. Es una interacción dinámica entre las condiciones externas de la vida de un individuo y la percepción interna de esas condiciones (Zhang \& Zhang, 2018)this research aims to clarify how leisure and tourism contribute to the elderly's quality of life (QOL. En función a la respuesta del adulto mayor, se obtuvo que el $61 \%$ tiene una mala calidad de vida, el $24 \%$ considera que es regular y el 14\% describió que es buena (Tabla 9); sin embargo, es necesario considerar a los adultos mayores con un nivel bajo.

Tabla 10.

Necesidades de las personas adultas mayores

\begin{tabular}{lcc}
\hline Necesidades & Frecuencia & $\%$ \\
\hline Obra social & 31 & $39 \%$ \\
\hline Fuentes de Empleo & 12 & $15 \%$ \\
\hline Apoyo económico & 6 & $7 \%$ \\
\hline Apoyo médico & 8 & $10 \%$ \\
\hline $\begin{array}{l}\text { Apoyo en la agricultura y } \\
\text { ganadería }\end{array}$ & 2 & $3 \%$ \\
\hline $\begin{array}{l}\text { Programas y proyectos en } \\
\text { beneficio común }\end{array}$ & 20 & $25 \%$ \\
\hline $\begin{array}{l}\text { Atención por las } \\
\text { discapacidades. }\end{array}$ & 1 & $1 \%$ \\
\hline TOTAL & 80 & $100 \%$ \\
\hline
\end{tabular}

Abordar las necesidades de atención y apoyo insatisfechas de una población que envejece, y diseñar servicios y soluciones centrados en lo que las personas mayores necesitan o quieren, se está convirtiendo en una prioridad urgente de salud pública. En consideración a lo mencionado, el $39 \%$ considera que es necesario obras sociales, el $25 \%$ programas y proyectos en beneficio común, $15 \%$ fuentes de empleo y el $10 \%$ apoyo médico, como se puede ver representado en la Tabla 10.

\section{Conclusiones}

En función a lo descrito, para lograr una vida digna, sobre todo en los adultos mayores, el Estado tiene la responsabilidad de brindar acciones viables que apruebe la inserción adecuada de los adultos mayores con el fin de proveer medios que permitan la inclusión, participación, protección social, prestación oportuna y de calidad en servicios de salud y demás servicios públicos, pensiones asistenciales, actividades lúdicas/recreativas para mejorar su atención, y 
jubilación universal; para ello se requiere el trabajo coordinado de todos los niveles de Gobierno conjuntamente con todas sus instituciones.

Está claro cuán necesario es el desarrollo de políticas y herramientas integradas, a través de la combinación de todos los aspectos que son fáciles de entender para los diferentes actores públicos y privados involucrados. Los resultados de este estudio podrían ser útiles para apoyar a los responsables políticos y técnicos en el desarrollo de futuros planes sectoriales.

Esta investigación tuvo varias limitaciones, dados los pocos datos disponibles y la sensibilidad temporal del brote pandémico, se direcciona únicamente al método elegido y las técnicas de muestreo. Por lo tanto, se puede decir que los participantes que fueron seleccionados, pueden no reflejar un verdadero modelo universal. Además, la salud psicológica, el aislamiento y el efecto pandémico informado se basan en las propias declaraciones de los participantes, y esto puede no ser coherente con una evaluación de los profesionales de la salud mental. 


\section{Referencias}

Acuña, M. (2021). América latina. Entre la nueva realidad y las viejas desigualdades. Telos Revista de Estudios Interdisciplinarios En Ciencias Sociales, 23(1), 129-140. https://doi.org/10.36390/telos231.10

De Pue, S., Gillebert, C., Dierckx, E., Vanderhasselt, M. A., De Raedt, R., \& Van den Bussche, E. (2021). The impact of the COVID-19 pandemic on wellbeing and cognitive functioning of older adults. Scientific Reports, 11(1), 1-11. https://doi.org/10.1038/s41598-021-84127-7

Downes, L., \& Brosseuk, D. (2021). The sophisticated literacy practitioner and the global pandemic. The Australian Educational Researcher, 1-19. https://doi.org/10.1007/s13384-021-00450-y

Flórez, L. (2020). Pandemia COVID-19: ¿Qué más puedo hacer? Revista de La Facultad de Medicina Humana, 20(2), 175-177. https://doi.org/10.25176/rfmh.v20i2.2941

García, L. (2021). COVID-19 y educación a distancia digital: preconfinamiento, confinamiento y posconfinamiento. Revista Iberoamericana de Educación a Distancia, 24(1), 9-25. https://doi.org/10.5944/ried.24.1.28080

Güner, R., Hasanoğlu, İ., \& Aktaş, F. (2020). Covid-19: Prevention and control measures in community. Turkish Journal of Medical Sciences, 50(SI-1), 571-577. https://doi.org/10.3906/sag-2004-146

Hernández-Sampieri, R., \& Mendoza, C. (2018). Metodología de la investigación (M. Rocha (ed.); Primera). Mc Graw Hill Education.

Instituto Nacional de Estadísticas y Censos. (2010). Población y Demografía. INEC. https://www.ecuadorencifras.gob.ec/censo-de-poblacion-y-vivienda/

Jaul, E., \& Barron, J. (2017). Age-Related Diseases and Clinical and Public Health Implications for the 85 Years Old and Over Population. Frontiers in Public Health, 5, 1-7. https://doi.org/10.3389/fpubh.2017.00335

Martínez, G. (2020). Calidad de vida del adulto mayor del cantón Tisaleo de la provincia de Tungurahua [Universidad Técnica de Ambato]. http://repositorio.ucsg.edu.ec/handle/3317/15672

McFarland, A., Waliczek, T., Zajicek, J., \& Lineberger, D. (2013). Eating habits and knowledge of nutrition in older adults: A comparison of gardeners and nongardeners. HortTechnology, 23(6), 843-848. https://doi. org/10.21273/horttech.23.6.843

Pak, A., Adegboye, O., Adekunle, A., Rahman, K., McBryde, E., \& Eisen, D. (2020). Economic Consequences of the COVID-19 Outbreak: the Need for Epidemic Preparedness. Frontiers in Public Health, 8(May), 1-4. https://doi.org/10.3389/fpubh.2020.00241 
Petrilli, C. (2020). Factors associated with hospitalization and critical illness among 4,103 patients with Covid-19 disease in New York City. MedRxiv. https://www.medrxiv.org/content/10.1101/2020.04.08.20057794v1.full. pdf+html

Robalino, E. (2018). Personas adultos mayores, derecho a una Vida Digna y Atención Prioritaria [Universidad Central del Ecuador]. file:///C:/Users/Usuario/Downloads/T-UCE-013-AB-262-2018.pdf

Rojo, M., \& Bonilla, D. (2020). COVID-19: La necesidad de un cambio de paradigma económico y social. CienciAmérica, 9(2), 77. https://doi.org/10.33210/ca.v9i2.288

Urtamo, A., Jyväkorpi, S., \& Strandberg, T. (2019). Definitions of successful ageing: A brief review of a multidimensional concept. Acta Biomedica, 90(2), 359-363. https://doi.org/10.23750/abm.v90i2.8376

Zhang, L., \& Zhang, J. (2018). Impacts of leisure and tourism on the elderly's quality of life in intimacy: A comparative study in Japan. Sustainability (Switzerland), 10(12). https://doi.org/10.3390/su10124861 
Copyright (c) 2022 María Cristina Espín Meléndez, Johana Estefanía Constante Lascano y Diego Francisco Granja Zurita

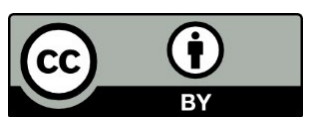

Este texto está protegido bajo una licencia internacional Creative Commons 4.0.

Usted es libre para Compartir-copiar y redistribuir el material en cualquier medio o formato - y Adaptar el documento - remezclar, transformar y crear a partir del material-para cualquier propósito, incluso para fines comerciales, siempre que cumpla las condiciones de Atribución. Usted debe dar crédito a la obra original de manera adecuada, proporcionar un enlace a la licencia, e indicar si se han realizado cambios. Puede hacerlo en cualquier forma razonable, pero no de forma tal que sugiera que tiene el apoyo del licenciante o lo recibe por el uso que hace de la obra.

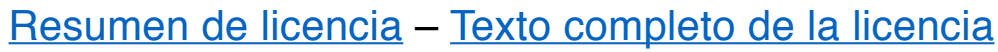

